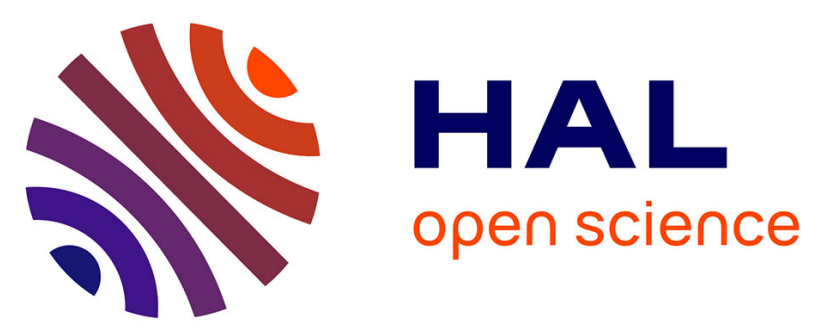

\title{
A new method for the evaluation of the end-to-end distance of the knee ligaments and popliteal complex during passive knee flexion
}

Goulven Rochcongar, Hélène Pillet, Elena Bergamini, S Moreau, Patricia Thoreux, Wafa Skalli, Philippe Rouch

\section{To cite this version:}

Goulven Rochcongar, Hélène Pillet, Elena Bergamini, S Moreau, Patricia Thoreux, et al.. A new method for the evaluation of the end-to-end distance of the knee ligaments and popliteal complex during passive knee flexion. The Knee, 2016, 23 (3), pp.420-425. 10.1016/j.knee.2016.02.003 . hal02470586

\section{HAL Id: hal-02470586 \\ https://hal.science/hal-02470586}

Submitted on 7 Feb 2020

HAL is a multi-disciplinary open access archive for the deposit and dissemination of scientific research documents, whether they are published or not. The documents may come from teaching and research institutions in France or abroad, or from public or private research centers.
L'archive ouverte pluridisciplinaire HAL, est destinée au dépôt et à la diffusion de documents scientifiques de niveau recherche, publiés ou non, émanant des établissements d'enseignement et de recherche français ou étrangers, des laboratoires publics ou privés. 


\title{
A new method for the evaluation of the end-to-end distance of the knee ligaments and popliteal complex during passive knee flexion
}

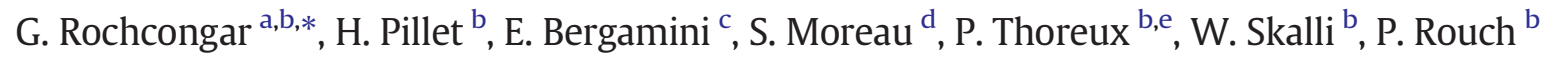 \\ a Département d'Orthopédie et Traumatologie, INSERM U1075 COMETE “Mobilité: Attention, Orientation É Chronobiologie”, Université de Caen, France \\ ${ }^{\mathrm{b}}$ Arts et Metiers ParisTech, LBM|Institut de Biomécanique Humaine Georges Charpak, Paris, France \\ ' Interuniversity Centre of Bioengineering of the Human Neuromusculoskeletal System, Department of Movement, Human and Health Sciences, University of Rome "Foro Italico", \\ P.zza Lauro de Bosis 15, 00135 Rome, Italy \\ ¿ Laboratoire d'Anatomie, Université de Caen, France \\ e Service de Chirurgie Orthopédique et Traumatologique, Hôpital Avicenne - Université Paris 13, Sorbonne Paris Cité, Bobigny, France
}

Keywords:

Ligament length

Knee kinematics

Popliteus tendon

Cruciate ligaments

Collateral ligaments

Ligament attachment sites

\begin{abstract}
A B S T R A C T
Background: Accurate knowledge about the length variation of the knee ligaments (ACL, PCL, MCL and LCL) and the popliteal complex during knee flexion/extension is essential for modelling and clinical applications. The aim of the present study is to provide this information by using an original technique able to faithfully reproduce the continuous passive knee flexion-extension kinematics and to reliably identify each ligament/tendon attachment site.

Methods: Twelve lower limbs (femur, tibia, fibula, patella) were tested and set in motion ( $\left.0-120^{\circ}\right)$ using an ad hoc rig. Tibio-femoral kinematics was obtained using an optoelectronic system. A 3D digital model of each bone was obtained using low-dosage stereoradiography. Knee specimens were dissected and the insertion of each ligament and popliteal complex were marked with radio opaque paint. ACL, PCL and MCL were separated into two bundles. Bone epiphyses were CT-scanned to obtain a digital model of each ligament insertion. Bones and attachment site models were registered and the end-to-end distance variation of each ligament/tendon was computed over knee flexion.

Results: A tibial internal rotation of $18^{\circ} \pm 4^{\circ}$ with respect to the femur was observed. The different bundles of the ACL, MCL and LCL shortened, whereas all bundles of the PCL lengthened. The popliteal complex was found to shorten until $30^{\circ}$ of knee flexion and then to lengthen.

Conclusion: The end-to-end distance variation of the knee ligaments and popliteal complex can be estimated during knee flexion using a robust and reliable method based on marking the ligaments/tendon insertions with radiopaque paint.

Level of evidence: Level IV
\end{abstract}

\section{Introduction}

The knee is one of the most studied joints with more than 110,000 occurrences in Pubmed. It is also probably the most complex one, characterised by a compromise between great stability and mobility. This is allowed by the interaction of different passive structures: femur, tibia, patella, ligaments, and menisci. Among them, the four major knee ligaments (anterior and posterior cruciate ligaments, ACL and PCL, medial and lateral collateral ligaments, MCL and LCL, respectively), together with the popliteal complex (comprising the femoral insertion of the popliteus tendon and the fibular insertions of the popliteo-fibular ligament [22]), play a crucial role in guiding the knee passive kinematics and stabilising this joint $[39,42]$. The knowledge of the biomechanical behaviour of these elements is essential to understand the complex kinematics of the healthy joint, and is an important prerequisite to understand ligament injury mechanisms, predict the consequences of ligament disruption, and properly design surgical interventions.

The variation of the length (commonly defined as the geometric distance between the ligament origin and insertion and hereinafter referred to as "end-to-end distance") of the major knee ligaments has been widely dealt with in the literature. However, most studies considered each ligament individually, with particular attention to the ACL $[1,16,44]$ or the PCL $[8,17,19,31]$ or, to a lesser extent, to the MCL $[33,41]$ or the LCL $[33,40,41]$. Few works studied the popliteal complex [40], although it has a critical role in the control of the rotation of the knee joint, especially limiting the external rotation of the tibia with respect to the femur [34]. Last but not least, methodological issues still exist related to the reliable identification of each ligament insertion [36] and, when ex-vivo studies are considered, to the reproduction of the knee passive 
kinematics, which are both crucial aspects when the accurate estimation of the ligament end-to-end distance is aimed.

To the authors' knowledge only two publications studied the end-toend distance variation of all the four major ligaments simultaneously during passive knee flexion ex-vivo, including the individual identification of the ligament attachment site locations [4,5]. In the first study, Belvedere et al. [4] used an optoelectronic system to identify the ligament attachment sites after minimal cadaver dissections and the position of each ligament insertion was digitised and expressed with respect to tibia and femur anatomical systems of reference by means of cluster of markers attached on a pointer and on the bones. However, although optoelectronic systems are considered as the gold-standard for the estimation of the knee kinematics, this is not the case for what concerns the ligament insertion identification. The above-mentioned procedure, in fact, apart from being time consuming, is affected by uncertainties due to difficulties for the surgeon to accurately palpate the ligament attachment sites without performing a complete dissection, particularly those of the medial and lateral collateral ligaments and of the PCL on the tibia. In the second study [5], a virtual palpation procedure was carried out to identify the ligament insertions on generic digital bone models. Subject-specific insertion locations were then estimated by matching the generic models to low-dose stereoradiography images of knee specimens. However, the accuracy of the ligament insertion identification was not assessed and no bundle separation was performed for the ACL, PCL and MCL. Moreover, none of these studies provided information about the insertion locations of the popliteal complex as well as about its end-to-end distance variation during knee flexion.

In the light of the above-mentioned considerations, the aim of the present study is to propose an original and robust technique to identify the knee ligaments and popliteal complex attachment sites and to describe how the end-to-end distance of these structures vary over passive knee flexion-extension movement.

\section{Methods}

\subsection{Specimens}

Twelve fresh frozen lower limbs were used in this study, six left and six right knees harvested from subjects aged between 47 and 79 years. Each specimen included entire femur with femoral head, patella, fibula and tibia without the ankle. Absence of trauma was checked and integrity of cartilage, meniscus and ligaments was inspected at the end of the experimentation during specimen dissection. Limbs were thawed at room temperature for $24 \mathrm{~h}$. Skin and muscles, except eight centimetres of quadriceps tendon and popliteus muscle, were removed before the study. Nine tantalum balls were placed into the metaphysis of each bone, three into the tibia, three into the femur and three into the patella.

\subsection{Kinematic data acquisition}

The specimens were set in motion using a device described and validated in previous literature [3] (Figure 1). The femur was rigidly fixed to a rig and the tibia was free to move (Figure 1). As no constraints have to be applied to coupled movements between the femur and the tibia, the device was slightly modified to allow free varus/valgus movements and a flexion-extension range of movement of $0^{\circ}-120^{\circ}$. A motor was connected to the tibial pilon by a rope and was used to pull the rope thus allowing the flexion-extension movement. A $10 \mathrm{~N}$ force was applied to the quadriceps tendon via a pulley hung in order to guide the patella. The direction of this force was as parallel as possible to the tendon itself. Clusters made of three retro-reflective markers each were screwed in the femur, the tibia and the patella (Figure 1). As different alignments between the femur and tibia could affect both the knee axial rotation and varus-valgus kinematics, special attention was paid

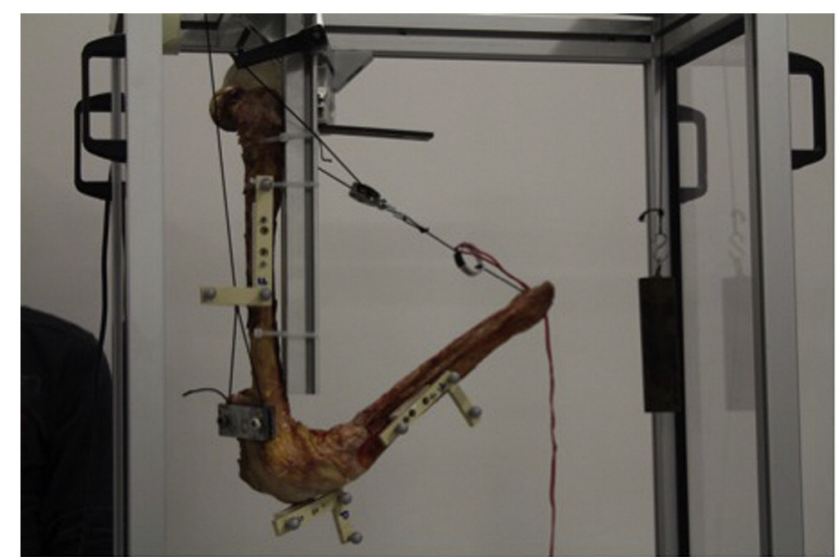

Figure 1. View of the device used to set in motion the knee specimens. The clusters of markers fixed to the femur, tibia and patella bones are also depicted.

to the correct alignment of the bones when mounting the specimens on the rig. The position and orientation of each cluster of markers were registered with an optoelectronic system previously used in motion analysis (Polaris, Northern Digital Inc., Canada, sampling frequency $=60$ samples/s) [7].

To obtain the three-dimensional (3D) tibio-femoral and patellofemoral kinematics, anatomical frames associated with each bone were defined. To this aim, two orthogonal digital radiographs of each knee specimen were simultaneously acquired using a low dosage X-ray system (EOS, EOS-imaging, France) and 3D digital models of the femur and tibia, with fibula and patella, were obtained through a reconstruction algorithm described in a previous study [5]. Femoral, tibial and patellar anatomical frames were defined following the indications reported by Schlatterer et al. [37]. The tantalum balls pierced in each bone, as well as the markers of each cluster, were also identified and the $3 \mathrm{D}$ coordinates of the centroid of each ball and marker with respect to the EOS system of reference were obtained. Technical frames associated with the marker clusters were defined allowing acquisition of a mathematical relation between anatomical and technical frames expected to be invariant due to the rigid body assumption. The bone models and the relevant anatomical axes were then registered with respect to the movement data obtained in the optoelectronic system of reference. The $3 \mathrm{D}$ kinematics of the tibio-femoral joint was estimated from the instantaneous position of the clusters of markers and flexion/extension, adduction/abduction and internal/external rotation angles were obtained using the Cardan convention and the sequence "ZXY". For each specimen and each angle, a similarity analysis was performed to investigate if significant differences existed in the kinematics obtained during the different cycles. To this aim, the Spearman correlation coefficient was calculated using IBM SPSS Statistics (IBM Corp., Armonk, NY, USA).

The kinematic variability during the six flexion-extension cycles was less than one degree and one millimetre with a high Spearman correlation ( $\mathrm{r}=0.98, \mathrm{p}<0.001$ for each knee). Therefore, as no significant hysteresis was present among the cycles, the average curve was considered as representative of the individual kinematics of each specimen. This confirmed the good reliability of the tested rig, which was previously reported by Azmy et al. for the patello-femoral joint [3], as well as the proper alignment of the bony segment during the kinematic acquisitions.

\subsection{Ligament attachment site identification and registration}

After kinematic data acquisition, knee specimens were fully dissected, according to anatomical references previously published $[12,13,22,38]$ in order to identify and mark ligaments/tendon origins and insertions using radio opaque paint composed of barium sulphate (Figure 2). The following structures were taken into account: ACL, PCL, 


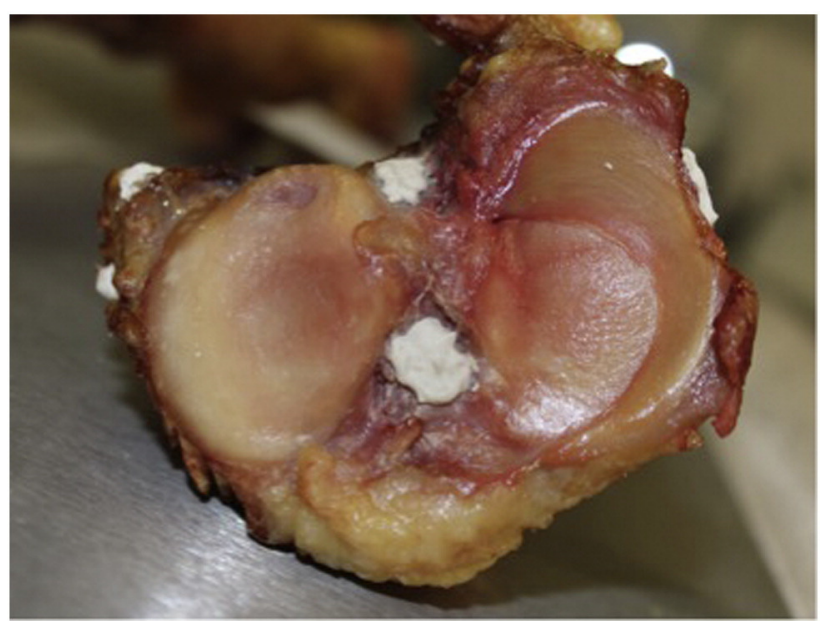

Figure 2. View of the proximal epiphysis of a randomly chosen tibia, showing the different ligament insertions marked with the radiopaque paint (white marks).

MCL (deep and superficial bundles, i.e. the two tibial insertions), LCL, and popliteal complex (going from the femoral insertion of the popliteus tendon to the fibular insertion of the popliteo-fibular ligament). For the sake of readability, the word "ligaments" will be used throughout the manuscript when referring to both the knee ligaments and the popliteal complex.

After the specimen was dissected and the attachment sites identified, the femoral and tibial epiphyses were scanned, using a computed tomography (CT) Scanner (Philips, Best, The Netherlands; thickness = $0.67 \mathrm{~mm}$, distance $=0.33 \mathrm{~mm}$ ). Digital models of each bone epiphysis, each ligament/tendon attachment site and each tantalum ball were obtained using Aviso ${ }^{\circledR}$ (Burlington, MA, US). The 3D coordinates of the tantalum balls with respect to the CT Scanner system of reference were then used to perform the registration between the CT Scan and the EOS systems of reference and, thus, to express the ligament attachment site coordinates with respect to the EOS frame. For those ligaments commonly separated into two bundles (ACL, PCL and MCL), the digital footprints obtained on each bone model were visually separated, using an ad hoc developed software, into two regions corresponding to the two bundles (antero-medial (AM) and postero-lateral (PL) for the ACL, antero-lateral (AL) and postero-medial (PM) for the PCL, superficial and deep for the femoral insertion of the $\mathrm{MCL}$ ). This procedure was carried out by an expert surgeon as described in the literature $[10,20,27]$. The centroid of each ligament attachment site was finally calculated for the whole insertions as well as for each bundle. Concerning the ACL and the PCL, the centroids of the whole insertions were referred to as "intermediate".

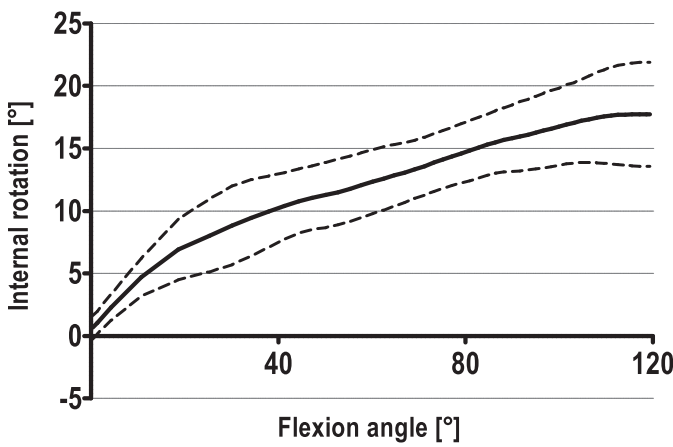

\subsection{Calculation of ligament end-to-end distance variations}

Knowing the position of each ligament insertion in the EOS reference frame, it was possible to obtain the same information in the clusterbased technical frame of each bone and to track the insertion positions all along the flexion-extension cycle. For each ligament (and bundle when considered), the distance between the femoral and tibial/fibular insertions was then estimated and referred to end-to-end distance. According to the existing literature $[14,15,18,23,24,32]$ and for the sake of readability, we referred to ligament lengthening when this distance increased and to ligament shortening when this distance decreased. The variation of the end-to-end distance of each ligament was then represented as a function of the tibio-femoral flexion-extension.

\section{Results}

For what concerns the knee kinematics, a tibio-femoral adduction of about $4^{\circ} \pm 4^{\circ}$ (mean \pm 1 standard deviation (SD)) and an internal rotation of the tibia with respect to the femur of about $18^{\circ} \pm 4^{\circ}$ at $120^{\circ}$ of flexion were observed (Figure 3 ).

The mean end-to-end distance of each ligament for a set of specific knee flexion angles is reported in Table 1. Figure 4 shows the ligament end-to-end distances expressed as a percentage of the maximal distance obtained during the knee movement (left panel) and the ligament end-to-end distance variations expressed in percentage with respect to the distance at knee maximal extension (right panel). During the knee flexion, the ACL (AM, PL and intermediary bundles), MCL (deep and superficial) and LCL shortened. The PCL lengthened of about $21 \%$. The popliteus complex shortened until $30^{\circ}$ of flexion and then lengthened.

\section{Discussion}

The present article is based on ex-vivo data and proposes a new methodology to identify the knee ligament attachment site locations in order to quantify the end-to-end distance variation patterns of the popliteal complex and of the main bundles of the four major knee ligaments ( $\mathrm{ACL}, \mathrm{PCL}, \mathrm{MCL}$, and $\mathrm{LCL}$ ) during continuous passive knee flexion. The proposed method proved to be robust and reliable and allowed obtaining information about the synergic behaviour of the main knee ligaments and of the popliteal complex, as well as about the individual 3D tibio-femoral kinematics.

Specifically, for what concerns the tibio-femoral kinematics, an internal rotation of the tibia of about $18^{\circ}$ at $120^{\circ}$ of flexion was found. An internal rotation was also reported by many authors between eight degrees and $29^{\circ}$ at $120^{\circ}$ of flexion $[25,29,42]$ and between $10^{\circ}$ and $15^{\circ}$ at $90^{\circ}$ of flexion $[5,30,43]$. Similarly, results about varus displacement are in agreement with previous studies which reported less than five degrees of adduction [5,43].

The results about the end-to-end distance of the ligaments in extended position are in general agreement with those reported in the existing literature. For what concerns the ACL, the end-to-end distances of the different bundles were found to be $35 \mathrm{~mm}$ (AM bundle), $32 \mathrm{~mm}$ (intermediate bundle), and $29 \mathrm{~mm}$ (PL bundle). Bicer et al. [6] reported

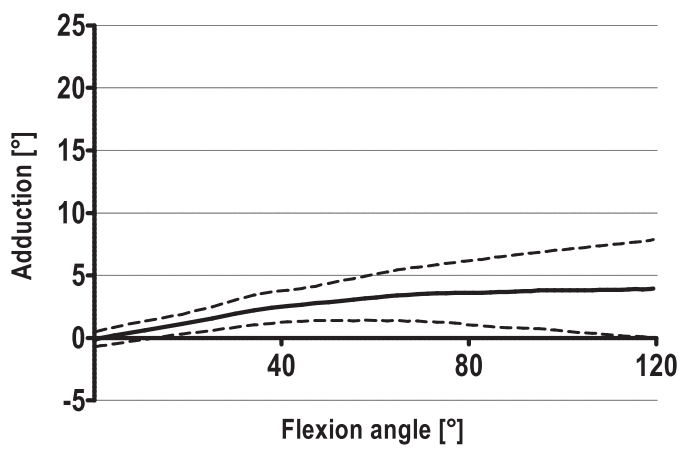

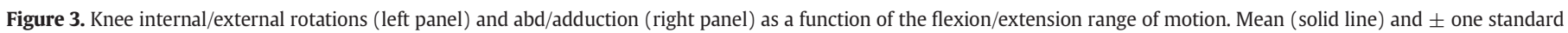
deviation (dotted line) are reported. 
Table 1

Mean \pm 1 one standard deviation of each ligament/tendon (and bundles where indicated) length $(\mathrm{mm})$ obtained at specific knee flexion angles.

\begin{tabular}{|c|c|c|c|c|c|c|}
\hline & $0^{\circ}$ & $15^{\circ}$ & $30^{\circ}$ & $60^{\circ}$ & $90^{\circ}$ & $120^{\circ}$ \\
\hline \multicolumn{7}{|l|}{ ACL } \\
\hline AM & $35 \pm 5$ & $35 \pm 4$ & $35 \pm 4$ & $34 \pm 4$ & $34 \pm 4$ & $33 \pm 4$ \\
\hline PL & $29 \pm 5$ & $28 \pm 5$ & $28 \pm 5$ & $26 \pm 4$ & $25 \pm 3$ & $25 \pm 3$ \\
\hline Intermediate & $32 \pm 4$ & $32 \pm 4$ & $32 \pm 4$ & $30 \pm 4$ & $29 \pm 4$ & $29 \pm 3$ \\
\hline \multicolumn{7}{|l|}{ PCL } \\
\hline $\mathrm{AL}$ & $27 \pm 3$ & $28 \pm 2$ & $28 \pm 3$ & $32 \pm 3$ & $34 \pm 3$ & $35 \pm 3$ \\
\hline PM & $34 \pm 5$ & $32 \pm 4$ & $32 \pm 3$ & $34 \pm 3$ & $35 \pm 3$ & $35 \pm 3$ \\
\hline Intermediate & $29 \pm 4$ & $30 \pm 3$ & $30 \pm 3$ & $33 \pm 3$ & $34 \pm 3$ & $35 \pm 3$ \\
\hline \multicolumn{7}{|l|}{ MCL } \\
\hline Deep & $36 \pm 4$ & $34 \pm 4$ & $34 \pm 4$ & $34 \pm 4$ & $34 \pm 4$ & $34 \pm 3$ \\
\hline Superficial & $92 \pm 9$ & $91 \pm 8$ & $91 \pm 8$ & $90 \pm 8$ & $89 \pm 8$ & $86 \pm 8$ \\
\hline $\mathrm{LCL}$ & $62 \pm 6$ & $60 \pm 6$ & $59 \pm 6$ & $57 \pm 6$ & $55 \pm 5$ & $53 \pm 5$ \\
\hline Popliteus & $44 \pm 5$ & $42 \pm 6$ & $41 \pm 6$ & $42 \pm 7$ & $44 \pm 7$ & $45 \pm 6$ \\
\hline
\end{tabular}

that the AM bundle is the longest with an end-to-end distance between $22 \mathrm{~mm}$ and $41 \mathrm{~mm}$ (mean value of about $32 \mathrm{~mm}$ ), whereas the PL bundle was shorter measuring $27 \mathrm{~mm}$. Regarding the PCL end-to-end distance, the results of the present study were $33 \mathrm{~mm}$ for the PM bundle, $29 \mathrm{~mm}$ for the intermediate bundle, and $27 \mathrm{~mm}$ for the AL bundle. Nakagawa et al. reported a PCL end-to-end distance up to $34 \mathrm{~mm}$ without considering bundle separation [31]. As for the end-to-end distances of the superficial and deep bundles of the MCL, results were respectively $92 \mathrm{~mm}$ and $36 \mathrm{~mm}$. These values are in agreement with a previous study of Liu et al. which reported an end-to-end distance of $95 \mathrm{~mm}$ for the superficial bundle of the MCL and $42 \mathrm{~mm}$ for the MCL deep bundle [26]. A very good concordance was observed for the LCL which was found to be $61 \mathrm{~mm}$. The same result was reported indeed by Diamantopoulos et al. [12]. Finally, the distance between the femoral insertion of the popliteus tendon and the fibular insertion of the popliteo-fibular ligament was found to be $43 \mathrm{~mm}$, in general agreement with a previous study published by Diamantopoulos et al. which reported a distance of about $35 \mathrm{~mm}$.

Many articles dealt with the variation of the end-to-end distance of each individual knee ligament during knee flexion-extension, both invivo and ex-vivo. In the early 90s, Amis and Dawkins found that the anteromedial bundle of the ACL was lengthening after $30^{\circ}$ of flexion, whereas the intermediary and posterolateral bundles were shortening [1]. Actually, recent in-vivo studies performed on unloaded knees concluded to a shortening of all bundles, the anteromedial bundle being the most isometric one $[18,44]$, as confirmed by the results of the present work. For what concerns the PCL, Nakagawa et al. and Jeong et al. reported a stretching over flexion for this ligament, especially for the anterolateral bundle $[17,31]$. This stretching, which was found to be about $20 \%$ with respect to the PCL end-to-end distance at knee full extension, is confirmed by the present results. As for the MCL, a shortening (six percent) of the deep bundle of the MCL was found in the present study, primarily during the first $40^{\circ}$ of flexion. Similarly, a shortening (six percent) of the MCL superficial bundle was found, occurring during all the flexion-extension range of motion. These findings are in agreement with a study of Park et al. concluding to a shortening of the medial part of the MCL superficial bundle [33]. Concerning the LCL, a shortening of $15 \%$ was found. This result confirms previous findings from Victor et al. [41] and Sugita and Amis [40] who reported that the LCL shortened by about $11 \%$.

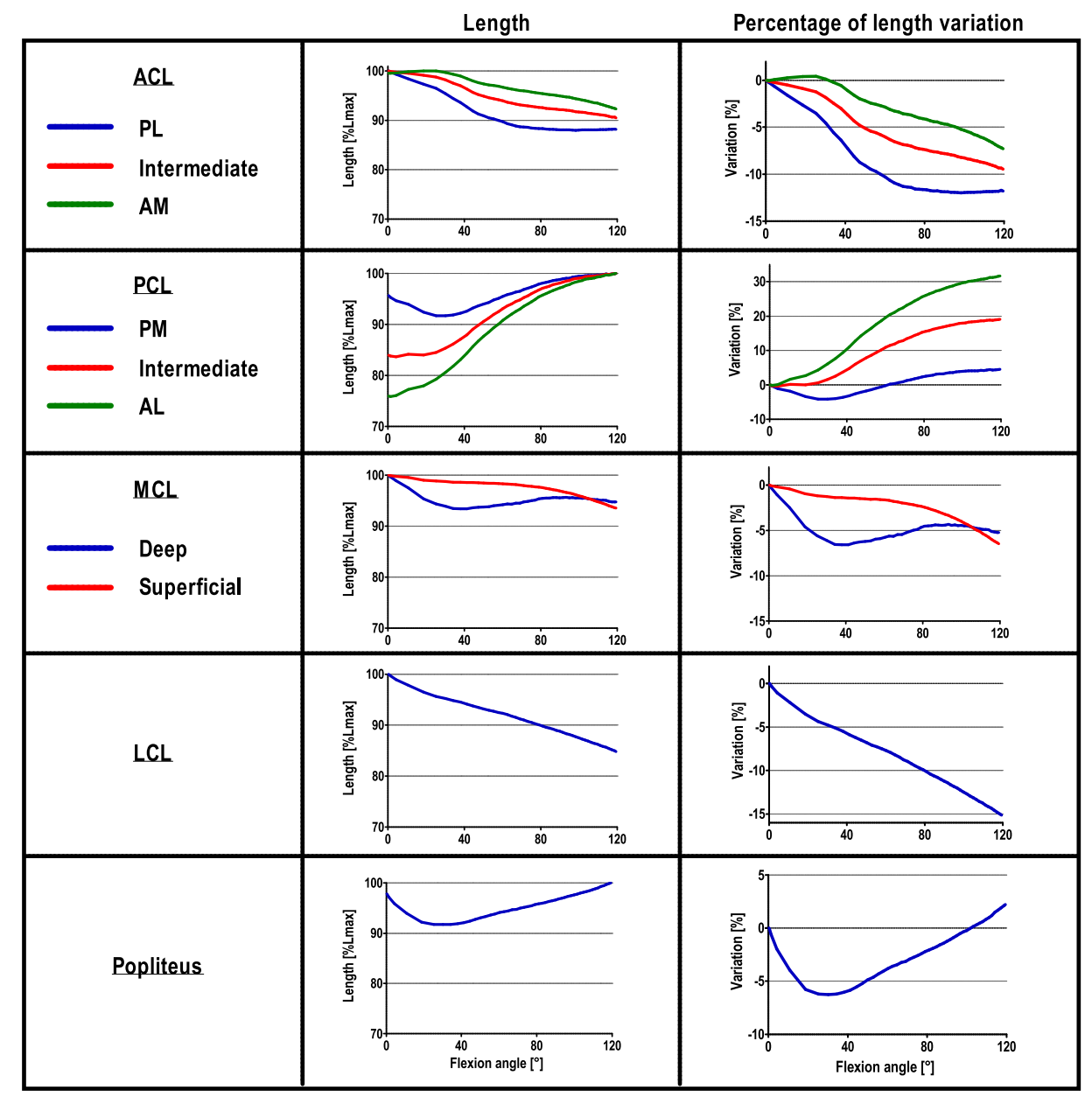

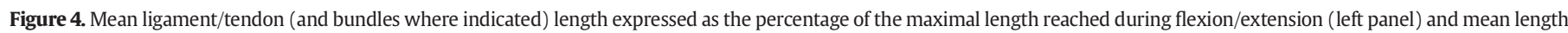
variation expressed as the percentage of the length reached at knee maximal extension (right panel). Both variables are depicted as a function of the knee flexion angle. 
According to the existing literature $[21,22,40]$ in the present study the femoral insertion of the popliteal tendon and the fibular insertion of the popliteo-fibular ligament were considered. In this respect, the quantification of the end-to-end distance variation of the popliteal complex is particularly interesting in clinical practice because many surgical procedures reconstruct the popliteus tendon using the abovementioned insertions $[2,11,45,46]$. When observing the end-to-end distance variation of the popliteal complex, it is worth noting that the inversion of the curve occurs around $30^{\circ}$ of knee flexion. A five percent shortening of the popliteal complex during the first $30^{\circ}$ of knee flexion was found, followed by a lengthening of about two percent in deep flexion. Sugita and Amis [40] found a lengthening of about $12 \%$. The limited sample size and large standard deviations $(10 \mathrm{~mm}$ corresponding to $25 \%$ of the global end-to-end distance of the complex) associated with the latter study could explain the difference in the reported results.

Only two papers described both the kinematics and the end-to-end distance variation of the knee ligaments considering all ligaments simultaneously $[4,5]$. However, these studies did not take the popliteal complex into account even if it plays a major role in controlling rotational stability of the knee. Moreover, minimal dissection together with an optoelectronic system [4] or virtual palpation procedures [5] were used to identify and register the ligament attachment sites, and therefore neither methodology is able to guarantee the realism required in clinical applications. Conversely, the original technique to identify the ligament attachment sites proposed in the present study allowed for a reliable and robust identification and registration of the insertions of the popliteal complex and of all the four major knee ligaments. This allowed for overcoming the main limitations related both to the use of optoelectronic systems and minimal dissection procedures [4] which do not allow accurate palpation of all ligament attachment sites, and to virtual palpation procedures [5] which do not necessarily take into account the accuracy and the variability of the ligament/tendon attachment site location.

Among the limitations of the present study, there is the fact that ligament length was modelled as the end-to-end distance between the ligament femoral and tibial/fibular insertions. Some ligaments, as the ACL and the MCL, are tightened in extension whereas others such as the PCL are slackened. Moreover, ligament wrapping around bone prominences and periosteal tissues was not accounted for. As ligaments are incompressible structures, their length cannot be less than the tension free length. This is particularly true for the PCL for which the end-to-end distance increases during the full range of motion whereas the "true" fibre lengthening occurs after $50^{\circ}$ of flexion [28]. It must be noted, however, that accurate modelling of the ligament path (and thus the quantification of the actual shortening and lengthening) is crucial when ligament forces/strains need to be assessed, which is not the case for the present study. Finally, the end-to-end distance approximation can be considered largely acceptable in most applications for which the data provided in the present paper could be useful for (i.e. subject-specific knee modelling for clinical or movement analysis purposes).

In this study, CT-scan was used to reconstruct only the accurate position of the ligament attachment sites, whereas the femur and tibia/fibula bone models were obtained using biplanar X-ray imaging. This is because the latter, being characterised by low-dosage irradiation and thus less risky for the subjects, can be used to obtain subject-specific information in-vivo even in routine clinical practice. Given the accuracy with which the bone models were obtained using the EOS system (on average lower than $1.3 \mathrm{~mm}$ as reported in Chaibi et al. [9] and Quijano et al. [35]), we consider the reconstruction errors to have no impact on the end results.

\section{Conclusion}

In the present study, an original technique was used for a reliable and robust identification of the insertions of the popliteal complex, and of the cruciate and collateral ligaments on knee specimens. Detailed information regarding the 3D tibio-femoral kinematics as well as regarding the end-to-end distance variation pattern of the considered structures and of their sub-bundles was provided. This information represents an important contribution towards the understanding of ligament injury mechanisms, the prediction of the consequences of ligament disruption, and hopefully the design of effective surgical interventions.

\section{Conflict of interest}

The authors declare that they have no conflict of interest for this publication.

\section{Acknowledgements}

Thanks to Ann-Laure Pollastri for her technical support and to Louis Dagneaux for helping during the experimental session. The authors also thank the ParisTech BiomecAM chair program on subject-specific musculoskeletal modelling.

This research was approved by the ethics committee of the human anatomy lab of the University of Caen (Basse Normandie, France).

\section{References}

[1] Amis A, Dawkins G. Functional anatomy of the anterior cruciate ligament. Fibre bundle actions related to ligament replacements and injuries. J Bone Joint Surg (Br) 1991;73-B:260-7.

[2] Arciero RA. Anatomic posterolateral corner knee reconstruction. Arthroscopy 2005; $21: 1147$.

[3] Azmy C, Guérard S, Bonnet X, Gabrielli F, Skalli W. EOS orthopaedic imaging system to study patellofemoral kinematics: assessment of uncertainty. Orthop Traumatol Surg Res 2010;96:28-36.

[4] Belvedere C, Ensini A, Feliciangeli A, Cenni F, D'Angeli V, Giannini S, et al. Geometrical changes of knee ligaments and patellar tendon during passive flexion. J Biomech 2012;45:1886-92.

[5] Bergamini E, Pillet H, Hausselle J, Thoreux P, Guerard S, Camomilla V, et al. Tibiofemoral joint constraints for bone pose estimation during movement using multibody optimization. Gait Posture 2011;33:706-11.

[6] Bicer EK, Lustig S, Servien E, Selmi TAS, Neyret P. Current knowledge in the anatomy of the human anterior cruciate ligament. Knee Surg Sports Traumatol Arthrosc 2010; 18:1075-84

[7] Billuart F, Devun L, Gagey O, Skalli W, Mitton D. 3D kinematics of the glenohumeral joint during abduction motion: an ex vivo study. Surg Radiol Anat 2007;29:291-5.

[8] Boisgard S, Levai JP, Saidane K, Geiger B, Landjerit B. Study of the posterior cruciate ligament using a 3D computer model: ligament biometry during flexion, application to surgical replacement of the ligament. Acta Orthop Belg 1999;65:492-502.

[9] Chaibi Y, Cresson T, Aubert B, Hausselle J, Neyret P, Hauger O, et al. Fast 3D reconstruction of the lower limb using a parametric model and statistical inferences and clinical measurements calculation from biplanar X-rays. Comput Methods Biomech Biomed Engin 2012;15:457-66.

[10] Colombet P, Robinson J, Christel P, Franceschi J, Djian P, Bellier G, et al. Morphology of anterior cruciate ligament attachments for anatomic reconstruction: a cadaveric dissection and radiographic study. J Arthrosc Relat Surg 2006;22:984-92.

[11] Coobs BR, Wijdicks CA. Armitage BM, Spiridonov SI, Westerhaus BD, Johansen S, et al. An in vitro analysis of an anatomical medial knee reconstruction. Am J Sports Med 2010;38:339-47.

[12] Diamantopoulos A, Tokis A, Tzurbakis M, Patsopoulos I, Georgoulis A. The posterolateral corner of the knee: evaluation under microsurgical dissection. J Arthrosc Relat Surg 2005;21:826-33.

[13] Edwards A, Bull AMJ, Amis AA. The attachments of the anteromedial and posterolateral fibre bundles of the anterior cruciate ligament. Knee Surg Sports Traumatol Arthrosc 2007; 15:1414-21.

[14] Hosseini A, Gill TJ, Li G. In vivo anterior cruciate ligament elongation in response to axial tibial loads. J Orthop Sci 2009;14:298-30.

[15] Hsieh Y, Draganich LF. Knee kinematics and ligament lengths during physiologic levels of isometric quadriceps loads. Knee 1997;4:145-54.

[16] Iwahashi T, Shino K, Nakata K, Nakamura N, Yamada Y, Yoshikawa H, et al. Assessment of the "functional length" of the three bundles of the anterior cruciate ligament. Knee Surg Sports Traumatol Arthrosc 2007;16:167-74.

[17] Jeong W-S, Yoo Y-S, Kim D-Y, Shetty NS, Smolinski P, Logishetty K, et al. An analysis of the posterior cruciate ligament isometric position using an in vivo 3-dimensional computed tomography-based knee joint model. J Arthrosc Relat Surg 2010;26: 1333-9.

[18] Jordan SS, DeFrate LE, Nha KW, Papannagari R, Gill TJ, Li G. The in vivo kinematics of the anteromedial and posterolateral bundles of the anterior cruciate ligament during weightbearing knee flexion. Am J Sports Med 2007;35:547-54

[19] King AJ, Deng Q Tyson R, Sharp JC, Matwiy J, Tomanek B, et al. In vivo open-bore MRI reveals region- and sub-arc-specific lengthening of the unloaded human posterior cruciate ligament. PLoS One 2012;7, e48714. 
[20] LaPrade RF, Engebretsen AH, Ly TV, Johansen S, Wentorf FA, Engebretsen L. The anatomy of the medial part of the knee. J Bone Joint Surg 2007;89:2000-10.

[21] LaPrade RF, Ly TV, Wentorf FA, Engebretsen L. The posterolateral attachments of the knee: a qualitative and quantitative morphologic analysis of the fibular collateral ligament, popliteus tendon, popliteofibular ligament, and lateral gastrocnemius tendon. Am J Sports Med 2003;31:854-60.

[22] LaPrade RF, Morgan PM, Wentorf FA, Johansen S, Engebretsen L. The anatomy of the posterior aspect of the knee. An anatomic study. J Bone Joint Surg 2007:89:758-64.

[23] Li G, Defrate LE, Rubash HE, Gill TJ. In vivo kinematics of the ACL during weightbearing knee flexion. J Orthop Res 2005;23:340-4.

[24] Li G, DeFrate LE, Sun H, Gill T]. In vivo elongation of the anterior cruciate ligament and posterior cruciate ligament during knee flexion. Am J Sports Med 2004;32: 1415-20.

[25] Li G, Zayontz S, DeFrate LE, Most E, Suggs JF, Rubash HE. Kinematics of the knee at high flexion angles: an in vitro investigation. J Orthop Res 2004;22:90-5.

[26] Liu F, Gadikota HR, Kozánek M, Hosseini A, Yue B, Gill TJ, et al. In vivo length patterns of the medial collateral ligament during the stance phase of gait. Knee Surg Sports Traumatol Arthrosc 2011;19:719-27.

[27] Lorenz S, Elser F, Brucker PU, Obst T, Imhoff AB. Radiological evaluation of the anterolateral and posteromedial bundle insertion sites of the posterior cruciate ligament. Knee Surg Sports Traumatol Arthrosc 2009;17:683-90.

[28] Markolf KL, Feeley BT, Tejwani SG, Martin DE, McAllister DR. Changes in knee laxity and ligament force after sectioning the posteromedial bundle of the posterior cruciate ligament. J Arthrosc Relat Surg 2006;22:1100-6.

[29] McPherson A, Kärrholm J, Pinskerova V, Sosna A, Martelli S. Imaging knee position using MRI, RSA/CT and 3D digitisation. Knee Mech Update Theor Exp Anal 2005. 38:263-8.

[30] Myers CA, Torry MR, Shelburne KB, Giphart JE, LaPrade RF, Woo SL-Y, et al. In vivo tibiofemoral kinematics during 4 functional tasks of increasing demand using biplane fluoroscopy. Am J Sports Med 2012;40:170-8.

[31] Nakagawa S, Johal P, Pinskerova V, Komatsu T, Sosna A, Williams A, et al. The posterior cruciate ligament during flexion of the normal knee. J Bone Joint Surg (Br) 2004; 86-B:450-6.

[32] Papannagari R, DeFrate LE, Nha KW, Moses JM, Moussa M, Gill TJ, et al. Function of posterior cruciate ligament bundles during in vivo knee flexion. Am J Sports Med 2007;35:1507-12.

[33] Park S, Defrate L, Suggs J, Gill T, Rubash H, Li G. The change in length of the medial and lateral collateral ligaments during in vivo knee flexion. Knee 2005;12:377-82.
[34] Pasque C, Noyes FR, Gibbons M, Levy M, Grood E. The role of the popliteofibular ligament and the tendon of popliteus in providing stability in the human knee. J Bone Joint Surg (Br) 2003;85-B:292-8.

[35] Quijano S, Serrurier A, Aubert B, Laporte S, Thoreux P, Skalli W. Three-dimensiona reconstruction of the lower limb from biplanar calibrated radiographs. Med Eng Phys 2013;35:1703-12.

[36] Rachmat HH, Janssen D, Zevenbergen WJ, Verkerke GJ, Diercks RL, Verdonschot N. Generating finite element models of the knee: how accurately can we determine ligament attachment sites from MRI scans? Med Eng Phys 2014;36:701-7.

[37] Schlatterer B, Suedhoff I, Bonnet X, Catonne Y, Maestro M, Skalli W. Skeletal landmarks for TKR implantations: evaluation of their accuracy using EOS imaging acquisition system. Orthop Traumatol Surg Res 2009;95:2-11.

[38] Siebold R, Schuhmacher P, Fernandez F, Śmigielski R, Fink C, Brehmer A, et al. Flat midsubstance of the anterior cruciate ligament with tibial "C"-shaped insertion site. Knee Surg Sports Traumatol Arthrosc 2014;23:3136-42.

[39] Southmayd W, Quigley TB. The forgotten popliteus muscle. Its usefulness in correction of anteromedial rotatory instability of the knee. A preliminary report. Clin Orthop 1978:218-22.

[40] Sugita T, Amis AA. Anatomic and biomechanical study of the lateral collateral and popliteofibular ligaments. Am J Sports Med 2001;29:466-72.

[41] Victor J, Wong PI, Witvrouw EP, Sloten JVI, Bellemans J. How isometric are the medial patellofemoral, superficial medial collateral, and lateral collateral ligaments of the knee? J Sports Med 2009;37:2028-36 (Oct. 2009).

[42] Wilson D. Ligaments and articular contact guide passive knee flexion. J Biomech 1998;31:1127-36.

[43] Wünschel M, Leichtle U, Lo J, Wülker N, Müller O. Differences in tibiofemoral kinematics between the unloaded robotic passive path and a weightbearing knee simulator. Orthop Rev 2012;4:e2.

[44] Yoo Y-S, Jeong W-S, Shetty NS, Ingham SJM, Smolinski P, Fu F. Changes in ACL length at different knee flexion angles: an in vivo biomechanical study. Knee Surg Sports Traumatol Arthrosc 2009;18:292-7.

[45] Zhang H, Feng H, Hong L, Wang X, Zhang J. Popliteofibular ligament reconstruction for posterolateral external rotation instability of the knee. Knee Surg Sports Traumatol Arthrosc 2009; 17:1070-7.

[46] Zorzi C, Alam M, Iacono V, Madonna V, Rosa D, Maffulli N. Combined PCL and PLC reconstruction in chronic posterolateral instability. Knee Surg Sports Traumatol Arthrosc 2013;21:1036-42. 